\title{
A Wireless and Batteryless Microsystem with Implantable Grid Electrode/3-Dimensional Probe Array for ECoG and Extracellular Neural Recording in Rats
}

\author{
Chih-Wei Chang ${ }^{1}$ and Jin-Chern Chiou ${ }^{2,3, *}$
}

1 Department of Bioengineering, University of California, Los Angeles, CA 90095, USA;

E-Mail: cw.chang@ucla.edu

2 Department of Electrical and Computer Engineering, National Chiao-Tung University, 1001 Ta Hseuh Rd., Hsinchu City 30010, Taiwan

3 Department of Medicine, China Medical University, No.91, Hsueh-Shih Road, Taichung City 40402, Taiwan

* Author to whom correspondence should be addressed; E-Mail: chiou@ mail.nctu.edu.tw; Tel.: +886-3573-1881; Fax: +886-3571-5998.

Received: 21 January 2013; in revised form: 21 March 2013 / Accepted: 28 March 2013 / Published: 8 April 2013

\begin{abstract}
This paper presents the design and implementation of an integrated wireless microsystem platform that provides the possibility to support versatile implantable neural sensing devices in free laboratory rats. Inductive coupled coils with low dropout regulator design allows true long-term recording without limitation of battery capacity. A 16-channel analog front end chip located on the headstage is designed for high channel account neural signal conditioning with low current consumption and noise. Two types of implantable electrodes including grid electrode and 3D probe array are also presented for brain surface recording and $3 \mathrm{D}$ biopotential acquisition in the implanted target volume of tissue. The overall system consumes less than $20 \mathrm{~mA}$ with small form factor, $3.9 \times 3.9 \mathrm{~cm}^{2}$ mainboard and $1.8 \times 3.4 \mathrm{~cm}^{2}$ headstage, is packaged into a backpack for rats. Practical in vivo recordings including auditory response, brain resection tissue and PZT-induced seizures recording demonstrate the correct function of the proposed microsystem. Presented achievements addressed the aforementioned properties by combining MEMS neural sensors, low-power circuit designs and commercial chips into system-level integration.
\end{abstract}

Keywords: microsystem; wireless; neural electrode array; biopotentials 


\section{Introduction}

Neuroscience engineering together with biomedical devices for neural recording in the central nervous system are widely viewed as a promising path to revolutionary progress in understanding neural functions and for the realization of practical neural prostheses. Also, long-tem, real-time and stable observation of the monitoring target for treating disorders such as seizures, as well as for rehabilitative prosthesis is required for these biomedical device designs that can simultaneously record neural signals from a large number of electrodes in awake animals [1]. Due to the small size of laboratory rats, a miniature, lightweight, wireless, implantable microsystem is the key solution to capture accurate biological signals from an untethered animal in its natural habitat $[2,3]$. Generally, wire connections are required for powering and data transmission. Use of wire connection between animals and instrumentation can cause serious problem, and even lead to the death of implanted animals when wire drag happens. Swivels are used to solve the dragging problem, but the area of movement is still limited and the price can be another problem. Additionally, channel numbers are limited and the implanted laboratory animal still cannot behave in their nature condition because of the wires. Benefitting from rapid developments in wireless communication, various wireless solutions including Zigbee [4] and Bluetooth [5], have been commercialized and applied in animal studies. Batteries are commonly used to eliminate the problem of power wires but they dominate the size and the cost of the implant device and become less attractive when the implant devices get smaller and one needs to occasionally replace their batteries [6]. Therefore, the demand for developing continuous long-term monitoring in free-behavior implanted animals is then highly desirable.

System-level integration provides efficient and low noise performance due to the compact size and shorter interconnections. Microsystem platforms with analog front ends, micro controller units and wireless data/power transferring ability offer the possibility to connect with all kind of neural sensing elements for different applications. Such microsystems provide amplification, filtering, digitization and wireless transmission for the raw signals collected from implantable sensors in the brain, and also lengthen implant life and avoid the wire plugs and additional size/weight required [7].

The majority of this paper is dedicated to the development of a biomedical microsystem platform associated with versatile implantable electrodes, that provides wireless and battery-less data and power transmission to/from an external host. A brief description with preliminary results has been presented in [8]. Two customized chips including a multichannel analog front end and a low dropout regulator are integrated with a commercial MCU and Bluetooth into a backpacked headstage-mainboard system. Also, two types of implantable electrodes, including a grid electrode and a 3D probe array, are presented for brain surface recording and 3D biopotential acquisition in the implanted target volume of tissue. In this paper, application examples will include auditory response measurement and seizure spike-wave discharge (SWD) monitoring in rats. The microsystem addressed the aforementioned properties by combining MEMS neural sensors, low-power circuit designs and commercial chips into system-level integration. 


\section{System Structure}

The presented microsystem comprises three typical elements: electrodes, connection cable and signal processing circuitry [9]. Figure 1 presents the system structure design and the application situation using the head-stage and main board in a backpack on a rat. The microelectrodes are implanted into the brain, connected to a connector and encapsulated with dental cement. A headstage with a 16-channel neural amplifier is then plugged onto the connector on the rat's head. The recorded signal from the electrodes is then amplified, filtered by the analog front end and output to the main board. Wires between the headstage and main board transferring output signals from the headstage and power/control signasl from the MCU are made from EEG lead wire for less noise sensitivity. A backpack is designed for rats to carry the main board, which consists of a microcontrol unit, Bluetooth transceiver, RF power receiving coil, rectifier and low dropout regulator. The external power transmission coils are located on the cage, driven by a power amplifier and a RF signal generator.

Figure 1. System structure of the presented microsystem.

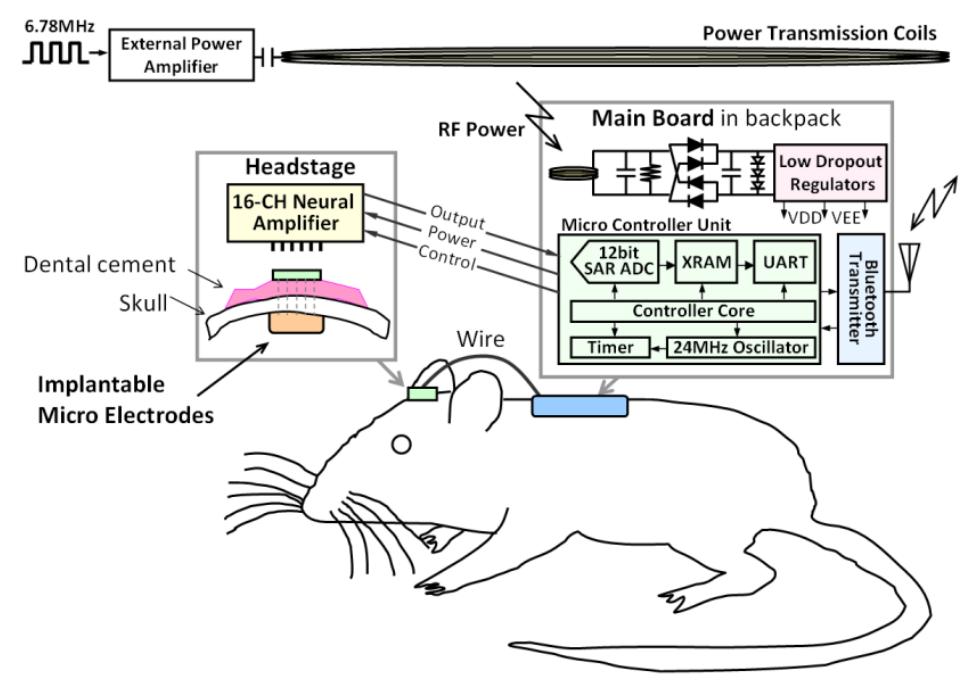

The main power loss in inductive power transmission is the electromagnetic (EM) wave reflection at the air-skin interface and heat dissipation effect inside the tissues [10]. Moreover, according to a prior study [11], skin tissue shows high absorption and reflection properties towards EM waves when the EM frequency is higher than $20 \mathrm{MHz}$ and lower than $1 \mathrm{MHz}$. In this paper, one of the industrial, scientific and medical (ISM) radio bands $(6.78 \mathrm{MHz})$ is chosen to meet the requirement of low tissue absorbability to avoid tissue damage.

\section{Implantable Sensing Devices}

\subsection{D Microprobe Array}

To access the full cell activity that originates in the target tissue, three dimensional distributed electrode arrays are required to achieve the recording and mapping of the neural signal network in the brain structure, which would be impossible to achieve by using 2-D planar arrays $[12,13]$. The stacking method for three-dimensional neural probe arrays creates 3-D probe arrays by assembling 2-D arrays 
and spacers layer by layer. As shown in Figure 2(A), for a $4 \times 4$ 3-D array, four 2-D arrays with four probes in each array and three spacers were required. Figure $2(B, C)$ shows microphotography images of the 2D arrays. Each 2-D array can be wire-bonded individually. Therefore, perpendicular bonding pads [14], folded structure with complex assembly [15,16] method are no longer needed. After the 2D parts were fabricated, a flip-chip bonder and thermosetting polymer were used in the assembly process. Convenient flip-chip technology was employed to accomplish the alignment, pressurization and heating process, while the thermosetting polymer provided an adhesive layer between two stacked layers. The thermosetting glue solidified at $185{ }^{\circ} \mathrm{C}$ in $180 \mathrm{~s}$ with an adhesive strength of $150-180 \mathrm{~kg} / \mathrm{cm}^{3}$. The maximal placement accuracy of the flip-chip was $0.5 \mu \mathrm{m}$ in a single bonding step. The average assembly time for a $4 \times 43 \mathrm{D}$ microprobe array by manual alignment was approximately 35 minutes, including heat curing time. Figure 2(D) shows the side view of wire bonding from each level of the array. Note that the thermosetting polymer layer is hidden between stacked layers therefore may not be visible. Figure 2(E) displays a photograph of the fabricated and assembled 3-dimensional probe array consisting of 16 silicon shafts with 3D distributed platinum recording sites on a one cent coin. The recording site is $100 \mu \mathrm{m}$ in diameter and narrow tip is about $23^{\circ}$.

Figure 2. Three dimensional neural probe array. (A) Stacking method (B,C) Close view of the electrode site and shafts (D) Wire bonding from each level of the array (E) Assembled array on one cent coin.
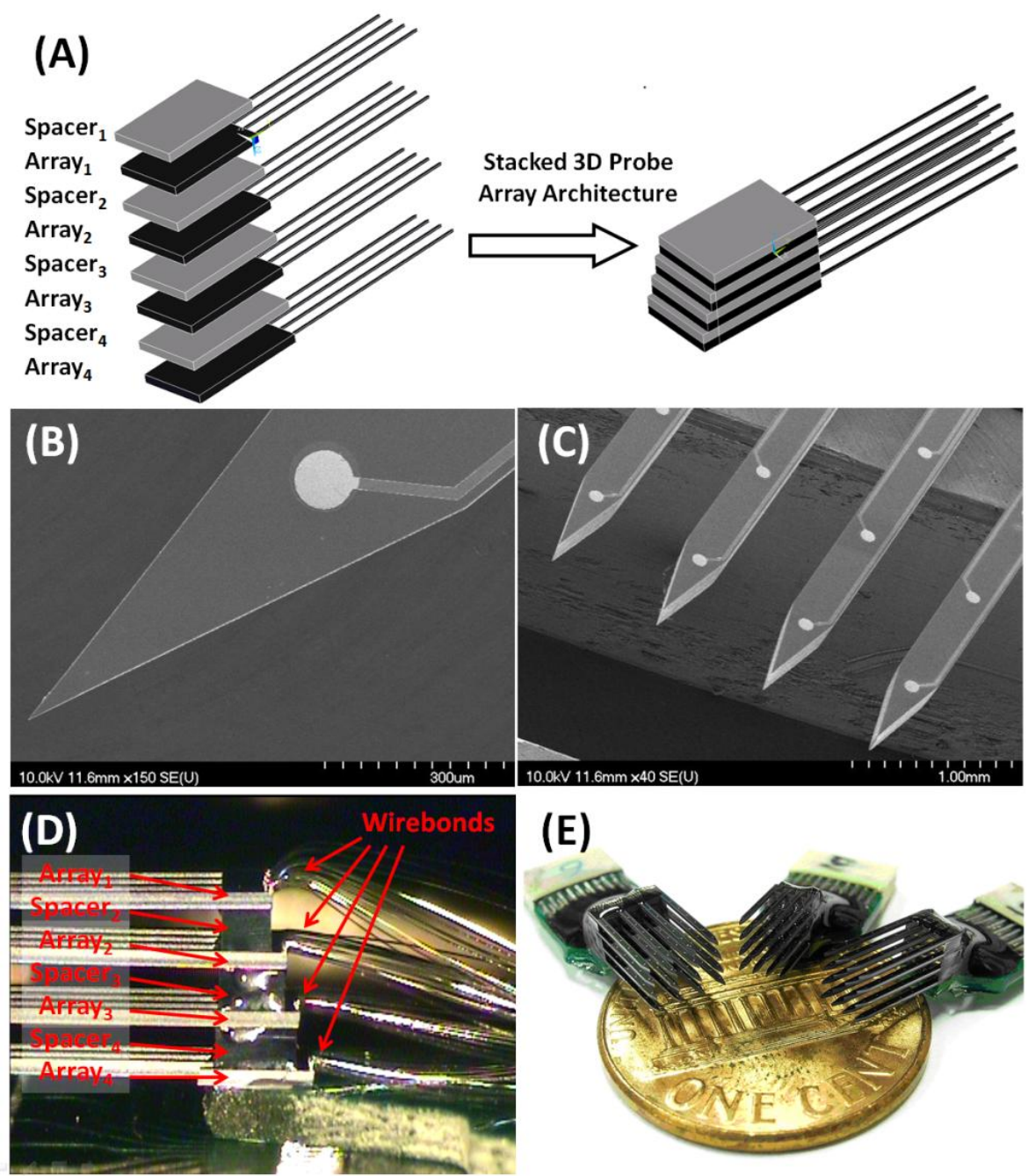

(E)

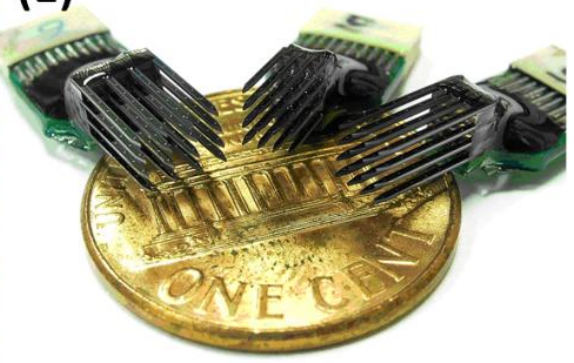




\subsection{Flexible Grid Electrode Array}

A flexible grid electrode array designed to be placed onto the brain cortex surface is proposed in this paper and acts as an implanted sensory device providing raw ECoG signals to the wireless microsystem. The design criteria of the grid electrode array include thickness, flexibility and bio-compatibility. Microstructure thickness allows minimal implantation damage [17]. Highly flexible structure properties are required to fit the curved brain surface. The presented grid electrode array is formed by $12 \mu \mathrm{m}$ thick Parylene-C as substrate with $500 \mu \mathrm{m}$ diameter platinum electrodes as sensing material. Benefitting from its flexibility properties, the grid electrode array can perfectly fit the brain surface curvature and collect the neural activity signals from the cortex surface.

Figure 3 illustrates the proposed fabrication process flow: (1) sacrificial layer deposition on the substrate; (2) Parylene-C deposition using CVD technique; (3) platinum electrode and interconnection wire definition using lift-off technique; (4) 2nd Parylene-C deposition; (5) hard mask patterning for grid electrode array shape definition; (6) dry etching Parylene-C; (7) structure release by washing the sacrificial layer material. Figure 3(B) shows a close-up view of the recording electrode array. Parylene-C shows its transparency propertiesy in Figure 3(C). The grid electrode array is packaged on a pre-designed PCB with a connector to be linked to the headstage. The grid array is $31.2 \mathrm{~mm}$ in length, $4.3 \mathrm{~mm}$ in width, and approximately $12 \mu \mathrm{m}$ in thickness. A total 1 of 16 platinum electrodes are fabricated with $500 \mu \mathrm{m}$ in diameter, $750 \mu \mathrm{m}$ in pitch. The width of the routing wire is $100 \mu \mathrm{m}$.

Figure 3. Flexible grid electrode array (A) Fabrication process. (B) Close-up view of the sensing electrodes. (C) Packaging and connection by a connector to the printed circuit board.

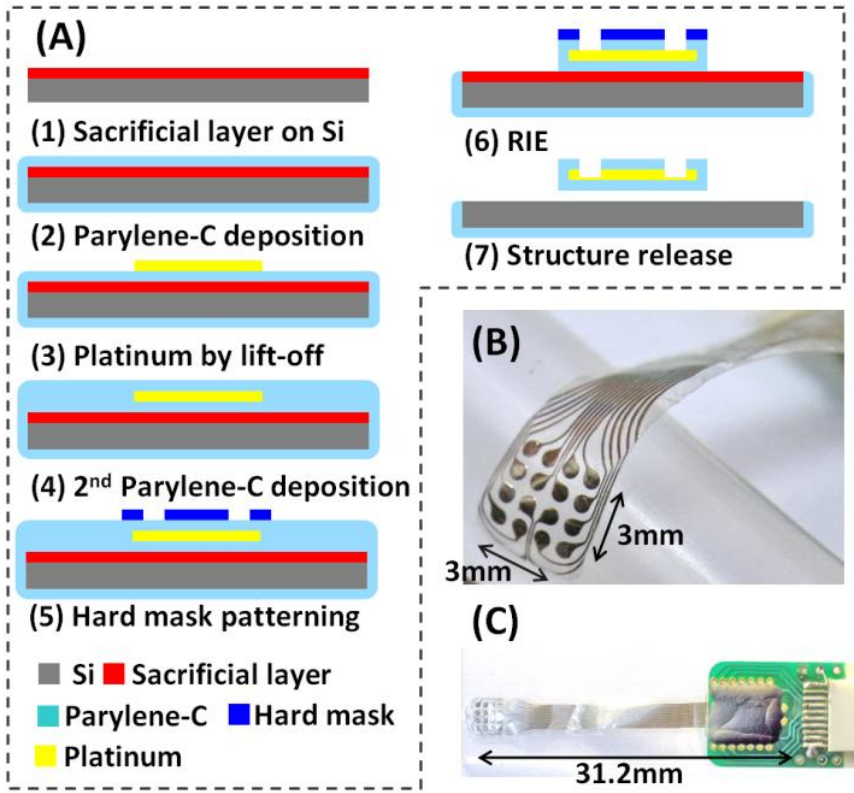

\section{Wireless and Batteryless Microsystem}

\subsection{RF Powering Electronics}

The RF-powering system block diagram is shown in the upper part of the main board in Figure 1. External RF power produced by a Class-E amplifier is coupled to the microsystem via a tuned LC 
network followed by a full-wave rectifier and LDO regulators to produce stable system and reference supplies. Low forward voltage Schottky diode and low equivalent series resistance (ESR) capacitance are selected for low ripple noise performance.

To date, the study of inductive coupled RF power transmitted by coils has been well established by following the electromagnetic induction of Faraday's Law [18]. Various approaches have been presented for optimal inductive coil design in implantable electronics [19-21]. In this paper, the transmission coils are designed to be located on the wall of the cage. The design procedure follows the method we used before and can also be found in [10,22-24]. The fabricated receiving and external coils made by 24/16 AWG copper wire are $1.5 \mathrm{~cm}$ and $22 \mathrm{~cm}$ in diameter. The inductive link exhibits pretty low coupling efficiency due to the large transmission coils design, but enough for powering the proposed microsystem. Efficient power coupling design in a large moving area for implanted animals will be our next step.

Figure 4(A) illustrates the block diagram of the low-dropout linear regulator. The architecture is modified from a typical low-dropout regulator topology with power MOSFET and OTP to enhance the driving current and avoid temperature damage to tissue [25]. The error amplifier amplifies the voltage difference between the reference voltage and divided load voltage and switches the power MOSFET (PMOS). Figure 4(B) shows the schematic of the error amplifier. MI27 is the power MOSFET illustrated in Figure 4(A). The over-temperature protection (OTP) design is used to avoid thermal damage to tissues due to the high temperature $\left(>40{ }^{\circ} \mathrm{C}\right)$ caused by the operating circuit. The OTP concept is realized by ROND resistor in tsmc $0.35 \mu \mathrm{m}$ process. Hysteresis functions when temperature is higher than $40{ }^{\circ} \mathrm{C}$, the power MOSFET turns off; when temperature drop down lower than $37^{\circ} \mathrm{C}$, OTP pull high to open the power MOSFET again. Figure 4(C) shows the optical microphotograph of the fabricated LDO regulator chip via a TSMC $0.35 \mu \mathrm{m} 2 \mathrm{P} 4 \mathrm{M}$ process. The die size is $1.42 \times 0.95 \mathrm{~mm}^{2}$.

Figure 4. (A) Block diagram of the LDO regulator (B) Schematic of error amplifier (C) Microphotograph of the fabricated LDO regulator chip.

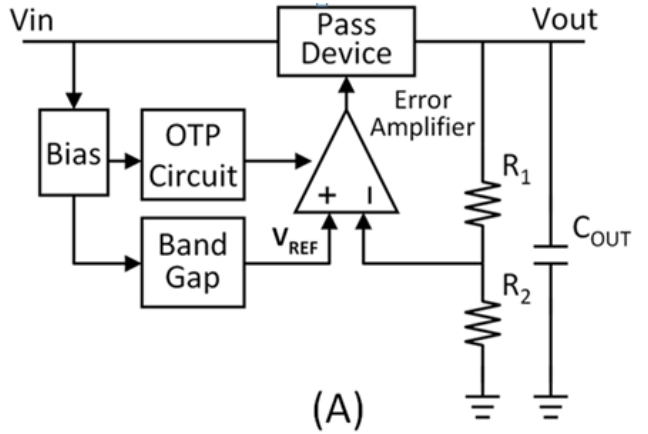

(A)

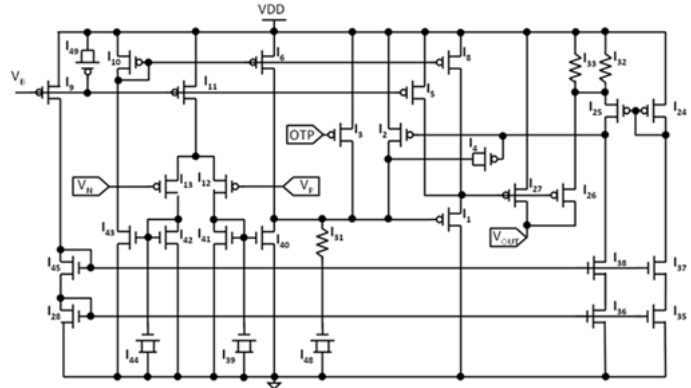

(B)

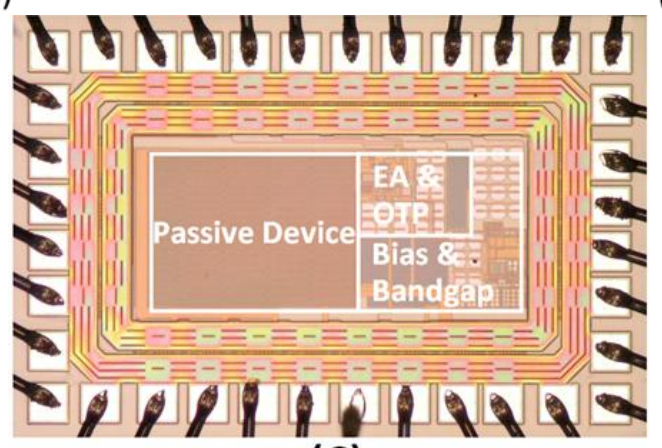

(C) 


\subsection{6-channel Analog Front End}

The 16-channel analog-front-end (AFE) amplifier provides signal conditioning and filtering of the weak neural potentials recorded by the electrode arrays. The block diagram of the complete 16-channel amplifier is shown in Figure 5(A). Differential difference amplifiers (DDA) are used as instrumentation stage of the AFE amplifier to meet our high-CMRR and low-noise requirements. Compared to other instrumentation amplifier designs, the DDA structure benefits from its high CMRR and device mismatches in the DDA design will solely influence the amplification gain. A 16:1 MUX controlled by the clock signal allows all 16 DDAs the share the same second and third amplifier/filter stages. The MUX frequency is set up to $200 \mathrm{kHz}$ to scan the 16-channel inputs. The second stage and third stage use operational transconductance amplifier (OTA) to implement low-pass filter, high-pass filter and gain amplifier with selectable bandwidth and gain. The gain and high frequency cut-off are controlled by a switchable capacitance array. The DDA used in this work is depicted in Figure 5(B). To reduce the flicker noise, the input stages are PMOS with wide width and operated in weak inversion region. The input stage transfers the input voltage into current. Common source amplifier is then transferring the current into differential input of the following two-stage amplifier. Finally, the DDA-based non-inverting amplifier is implemented from the topological placement of R1 and R2.

Figure 5. (A) Block diagram of the 16-channel amplifier, (B) Schematic of the differential difference amplifier, (C) Microphotograph of fabricated neural amplifier chip.

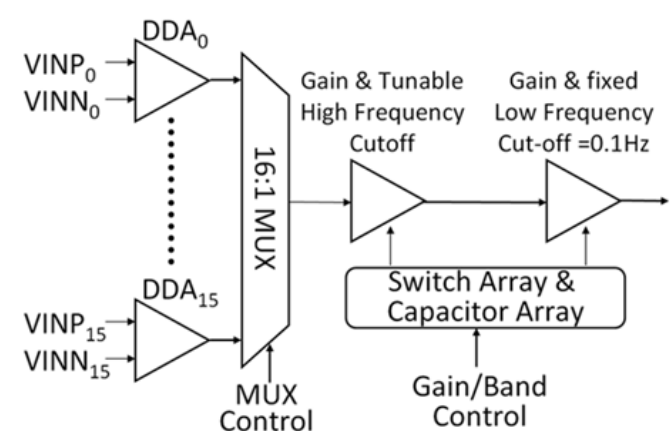

(A)

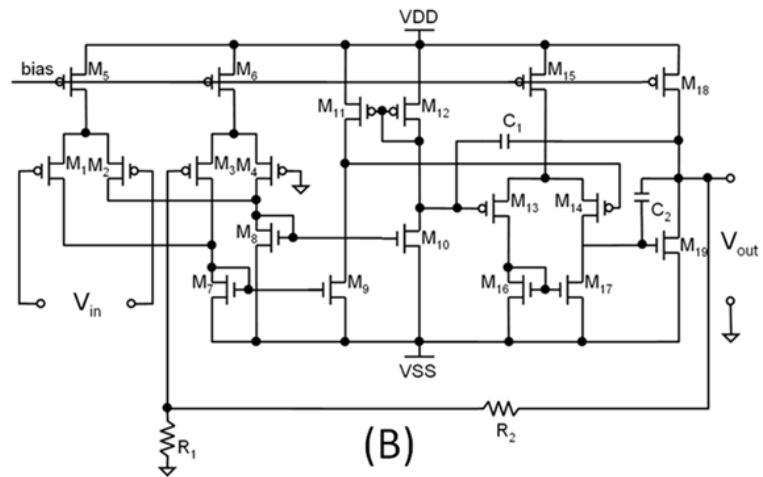

(B)

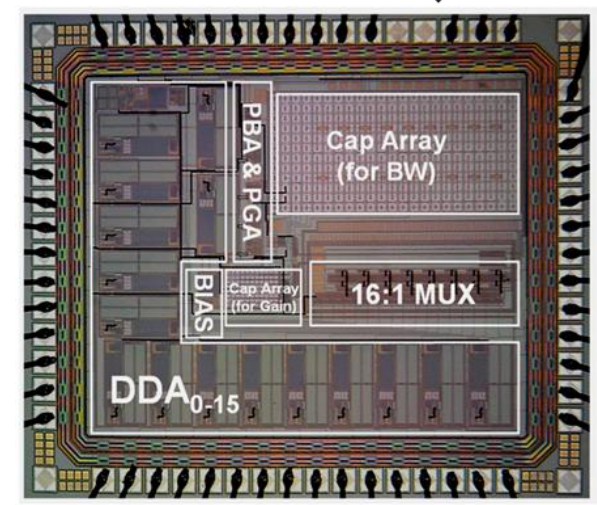

(C)

The 16-channel neural amplifier is fabricated using the TSMC $0.35 \mu \mathrm{m} 2 \mathrm{P} 4 \mathrm{M}$ CMOS process. A microphotograph of the complete chip is shown in Figure 5(C). The whole chip has a size of $4.18 \mathrm{~mm}^{2}$, including pads. 


\subsection{Integrated Microsystem}

The fabricated AFE chip is integrated onto the headstage by Chip-on-Broad (COB) technique with other associated passive components. The headstage is $3.4 \mathrm{~cm} \times 1.8 \mathrm{~cm}$ in size and weighs $3.92 \mathrm{~g}$. A commercial controller unit, Bluetooth wireless transceiver and the RF powering sub-circuit were integrated as the main board. A 4-layer PCB design is utilized with two powering layers in the middle layers. Figure 6 shows the picture of the fabricated microsystem including the headstage and main board. Note that the RF power receiving module is designed to be attached in the backside of the main board. The LDO regulators are located on the backside of the RF power receiving module which was illustrated in [8].

Figure 6. Fabricated microsystem including headstage and main board.

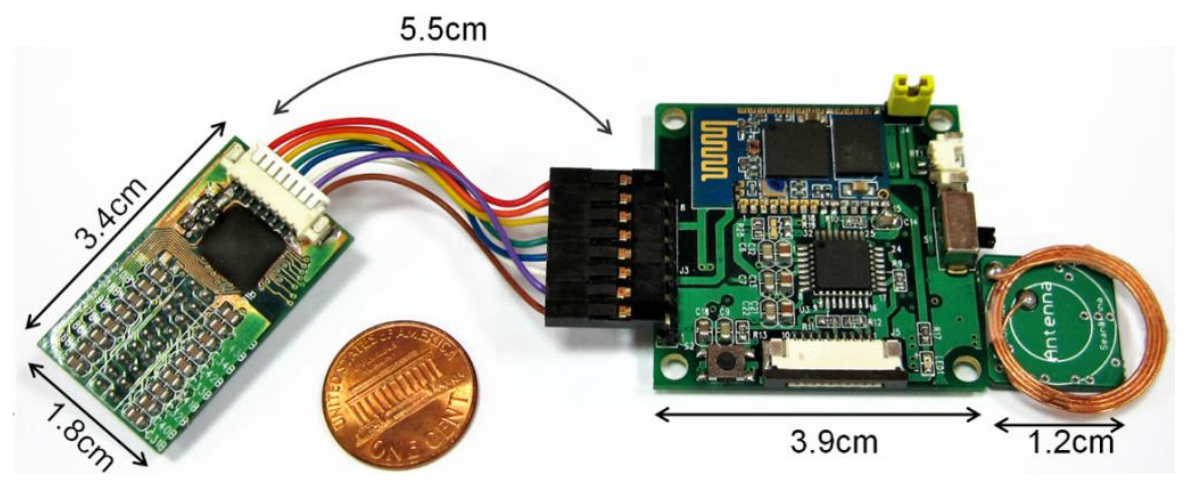

The front side of the main board consists of an MCU (C8051F58, Sliconlab Inc.) and wireless Bluetooth transceiver (BTM-192, Rayson Inc.). The main board is $39 \mathrm{~mm} \times 39 \mathrm{~mm}$ in size, and weighs $9.35 \mathrm{~g}$ including the RF power receiving module.

The presented LDO regulator provides $3.3 \mathrm{~V}, 1.8 \mathrm{~V}$ and $0.7 \mathrm{~V}$ for the ESD circuit, system VDD and reference bias, respectively. Also, an analog-digital DC voltage isolation technique including use of the middle two layers of the PCB as analog ground and digital ground, and magnetic beads used for connection between the analog and digital ground is utilized to isolate the digital noise coupling to the analog circuitry. Additionally, programming ports, power on LED, reset button and debugging circuitry are embedded on the board for function verification, although this can seriously increase the system dimensions. Detailed system specifications are summarized in Table 1.

Table 1. Summary of measured specifications.

\begin{tabular}{|c|c|c|c|}
\hline \multirow{10}{*}{ Head tage } & \multirow{8}{*}{$\begin{array}{l}\vec{a} \\
\hat{1} \\
\mathbf{1} \\
0 \\
0 \\
0 \\
0 \\
0 \\
0\end{array}$} & Gain & Tunable $52-75 \mathrm{~dB}$ \\
\hline & & Band & Tunable $0.1-6 \mathrm{KHz}$ \\
\hline & & CMRR & $>90 \mathrm{~dB}$ \\
\hline & & Current & $4.5 \mu \mathrm{A}$ per channel \\
\hline & & Supply Voltage & $1.8 \mathrm{~V}$ \\
\hline & & PSRR & $78 \mathrm{~dB}$ \\
\hline & & Input referred noise & $2 \mu \mathrm{Vrms}$ \\
\hline & & Chip area & $4.18 \mathrm{~mm}^{2}$ \\
\hline & \multicolumn{2}{|l|}{ Weight } & $3.92 \mathrm{~g}$ \\
\hline & \multicolumn{2}{|l|}{ Size } & $1.8 \times 3.4 \mathrm{~cm}^{2}$ \\
\hline
\end{tabular}


Table 1. Cont.

\begin{tabular}{|c|c|c|c|}
\hline \multirow{18}{*}{$\begin{array}{l}\text { Main } \\
\text { Board }\end{array}$} & \multirow{9}{*}{ 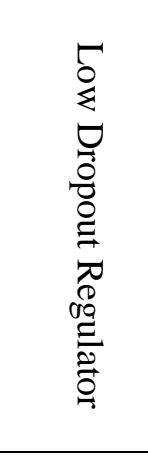 } & Output Voltage & $1.8-3.3 \mathrm{~V}$ \\
\hline & & Line Regulation & $4.5 \mathrm{mV}$ \\
\hline & & Load Regulation & $5 \mathrm{mV}$ \\
\hline & & Current Efficiency & $99 \%$ \\
\hline & & Output Current & $0 \sim 200 \mathrm{~mA}$ \\
\hline & & Quiescent Current & $45 \mu \mathrm{A}$ \\
\hline & & Current Limit & $252 \mathrm{~mA}$ \\
\hline & & Temperature Raise & $<2{ }^{\circ} \mathrm{C} @ 200 \mathrm{~mA}$ \\
\hline & & PSRR & $71 \mathrm{~dB} @ 10 \mathrm{~K} \mathrm{~Hz}$ \\
\hline & \multirow{4}{*}{$\frac{3}{2}$} & ADC Resolution & 12 bit \\
\hline & & Sample Rate & $1.25 \mathrm{kHz}$ per channel \\
\hline & & Current & $200 \mu \mathrm{A}$ \\
\hline & & Supply Voltage & $1.8 \mathrm{~V}$ \\
\hline & \multirow{3}{*}{ 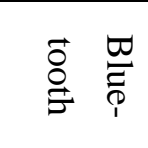 } & Transmission rate & Max 961,200 bps \\
\hline & & Current & $15 \mathrm{~mA}$ \\
\hline & & Supply Voltage & $1.8 \mathrm{~V}$ \\
\hline & \multicolumn{2}{|l|}{ Weight } & $9.35 \mathrm{~g}$ \\
\hline & \multicolumn{2}{|l|}{ Size } & $3.9 \times 3.9 \mathrm{~cm}^{2}$ \\
\hline \multirow{2}{*}{$\begin{array}{l}\text { Overall } \\
\text { System }\end{array}$} & \multicolumn{2}{|c|}{ Weight } & $13.17 \mathrm{~g}$ \\
\hline & \multicolumn{2}{|c|}{ Current Consumption } & $<20 \mathrm{~mA}$ \\
\hline
\end{tabular}

The MCUis 8051 core based micro controller with embedded $200 \mathrm{ksps}$ 12-bit SAR ADC, $50 \mathrm{MHz}$ oscillator, operates under $1.8 \mathrm{~V}$. A UART port is used to communicate with the Bluetooth module, which consists of a CSR Bluecore-4 single chip, complete $2.4 \mathrm{GHz}$ radio transceiver and print circuit antenna, designed for wireless connection. Benefitting from the easy usage of Bluetooth, a single laptop computer is utilized as transceiver host.

Figure 7. (A) Control flow of the MCU (B) Data timing and packaging method.

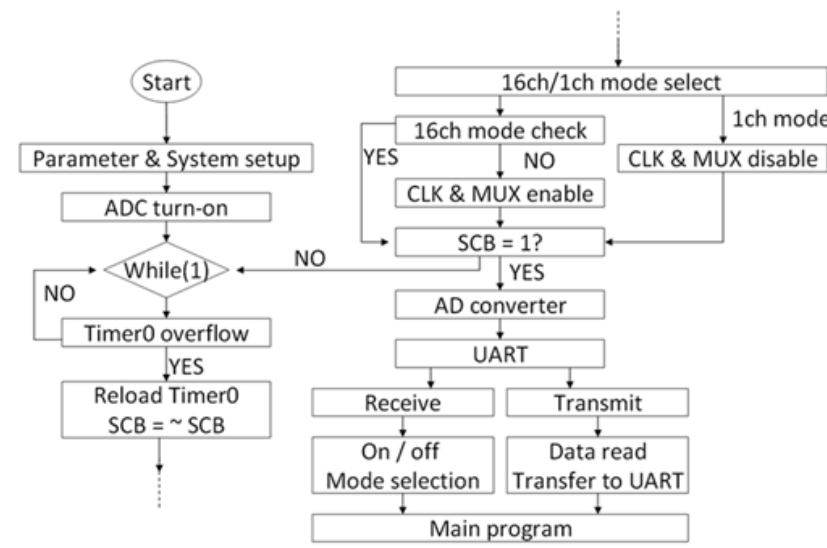

(A)

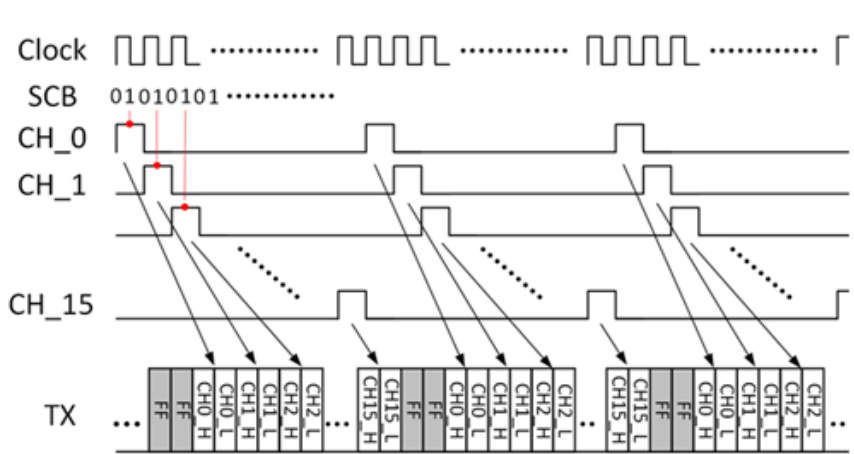

(B)

Figure 7(A) shows the control flow of the MCU, and detailed timing chart of the data is in Figure 7(B). The microsystem provides two operation modes, namely 16-channel mode and single channel mode. In the case of 16-channel mode, the clock signal generated by the MCU is sent to the AFE amplifier to scan the 16 channels and output the series data. A counter controlled 
Sample-control-Bit (SCB) is used to sample the analog data to ADC at the falling edge of the clock. Digitalized 12-bit data is then divided into first 8-bit and later 4-bit (2 bytes) because the UART only transfers 8-bit data in one time. After 16 channels are sent, two FF which acts marker are sent into UART before next 16-channel data. The marker is used by the software to divide and reconstruct the serial data back to simultaneous 16 channels. Windows-based software is designed for receiving data from the presented microsystem and sends commands to turn on, reset or parameter setting to the microsystem.

\section{Experimental Results}

Impedance spectroscopy was used to evaluate the impedance performance of the electrode- tissue interface. When the electrode sites come into contact with tissue, electrode-tissue interface impedance was established. High interface impedance will cause signal attenuation and induce considerable thermal noise while recording. Figure 8(A) displays the impedance spectrum of the grid electrode and 3-dimensional probe array in physiological saline solution, which is used to simulate the recording conditions. Result shows about $10 \mathrm{k} \Omega$ impedance at $1 \mathrm{kHz}$. The grid electrodes have lower impedance due to the larger electrode area.

Figure 8. (A) Impedance spectrum (B) Temperature raise of regulators (C) Tunable Gain/Band (D) PSRR performance of the LDO regulator.
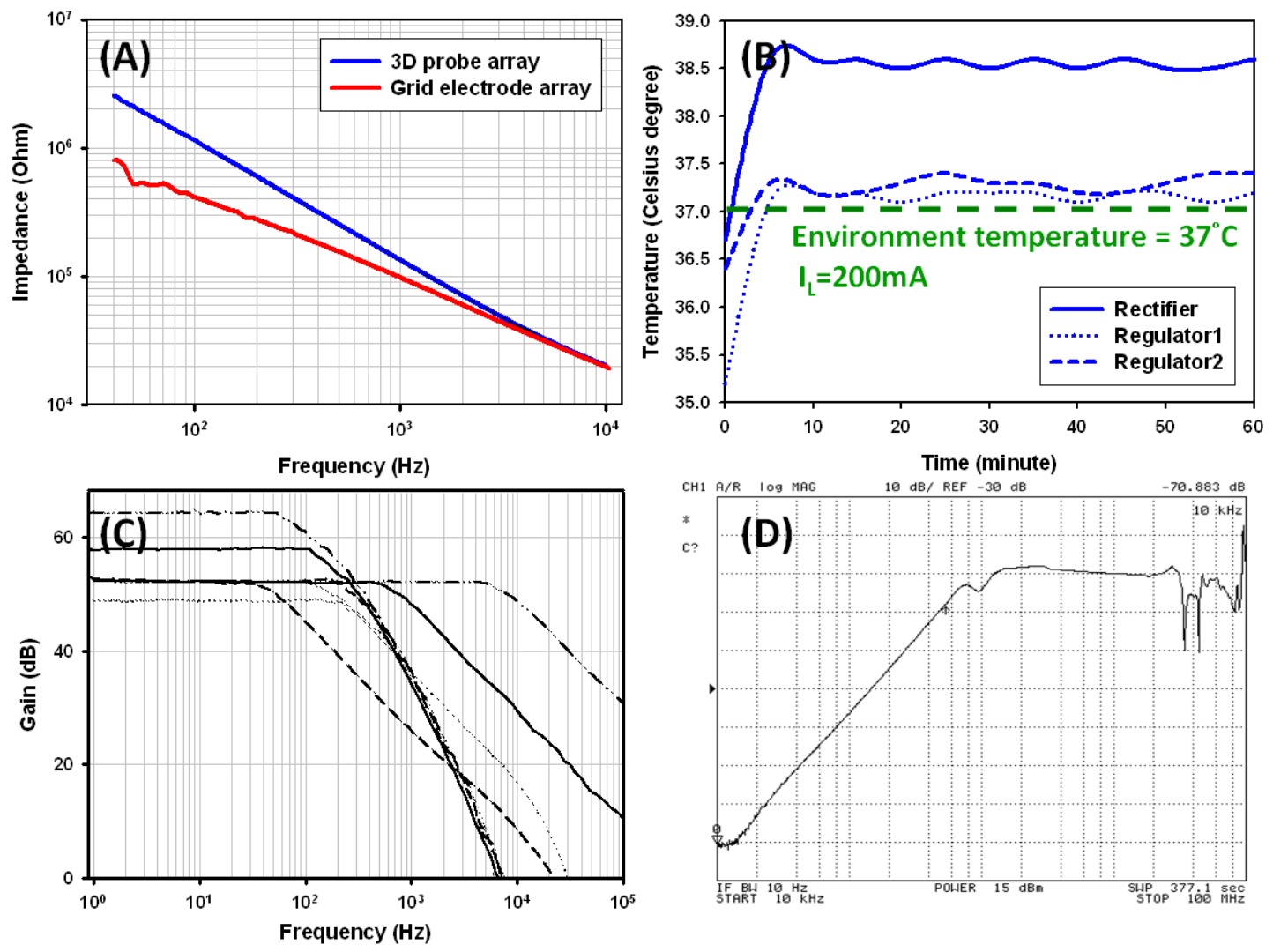

Figure 8(B) illustrates the temperature raise test of the wireless powering module under $37{ }^{\circ} \mathrm{C}$ environmental temperature, which is used to simulate the practical implant environmental conditions with maximal output current $(200 \mathrm{~mA})$ for $1 \mathrm{~h}$. The powering module was put into an oven heated to $37{ }^{\circ} \mathrm{C}$ to simulate the human body temperature. As shown in Figure 8(B), the raise of chip temperature 
follows the oven temperature, showing that less than $2{ }^{\circ} \mathrm{C}$ raise is achieved to meet the implantation requirements $[13,26]$. The LDO regulator also achieves low Quiescent-Current at $45 \mu \mathrm{A}$ and high loading current 0-200 mA capability with thermal protection. Tunable gain/band property of the 16-ch AFE amplifier is shown in Figure 8(C). Figure 8(D) is the test result of the power supply rejection ratio. The measured PSRR is around $70.883 \mathrm{~dB}$ at $10 \mathrm{kHz}$, indicating that it shows less PSRR than the simulation result due to parasitic inductance existing in the output ceramic capacitance, which decreases the PSRR performance.

To demonstrate the functionality of the fabricated gird electrode array, in vivo experiments on auditory stimulation response recording are induced. When an anesthetized rat receives specialized frequency/magnitude sound stimulation, an electrical response can be observed on a localized auditory area of the cortex. When the sound stimulation is received by the ears, it is transformed into neural signals and sent into brain which causes neural activities appearing in the auditory sensory area. By using the flexible grid electrode array, the area of interest of the brain is covered and recorded. Therefore, a localized electrical response spot could be detected and characterized for functional mapping and event evaluation. Practical implantation optical photograph is presented in Figure 9(A), which shows how the flexibility of the system allows excellent fitting to the exposed brain cortex. Figure 9(B) shows the close-up view of the electrode array contact area.

Figure 9. (A,B) Optical photograph of the grid electrode implantation (C) Time-magnitude plot of the averaged ECoG response under $9 \mathrm{kHz} 75 \mathrm{~dB}$ SPL stimulation.
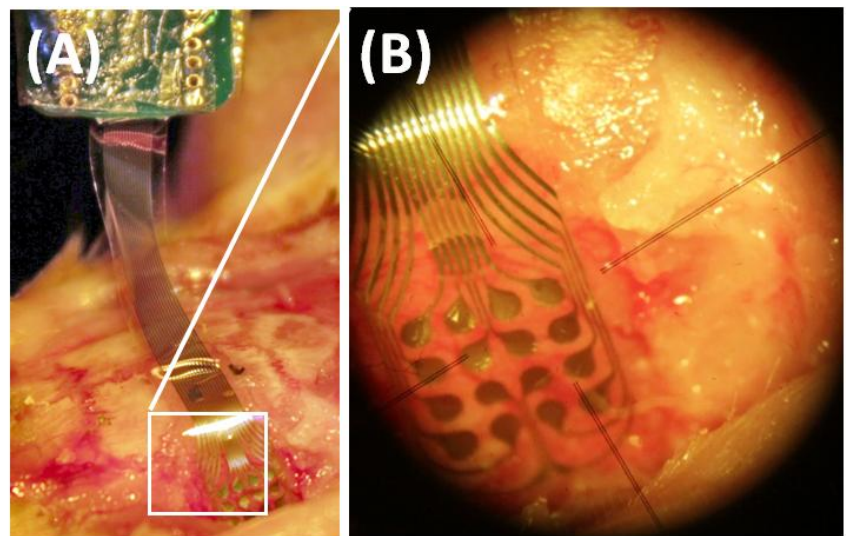

(C)
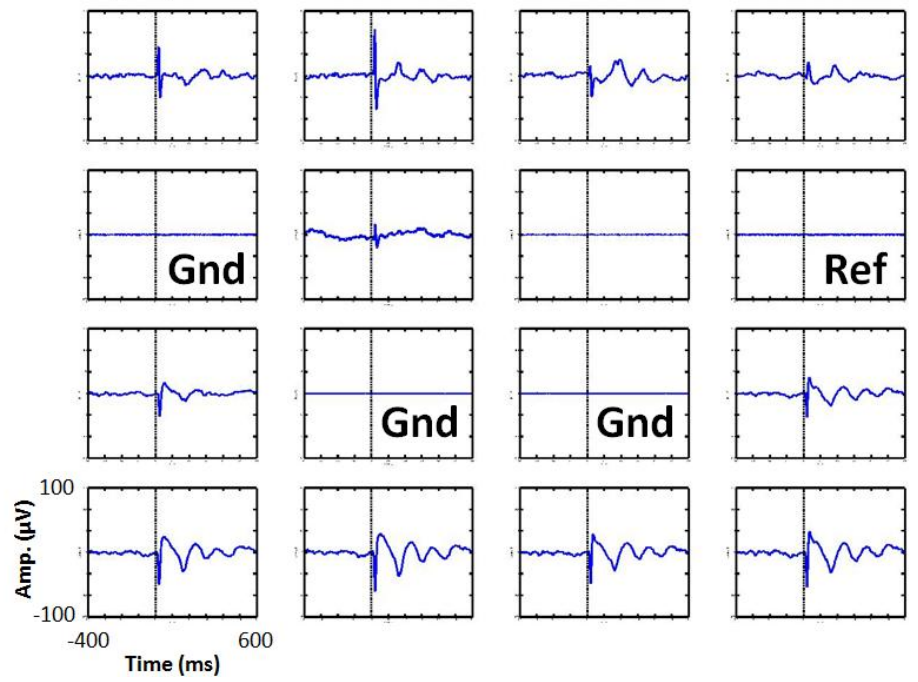
Figure 9(C) shows the auditory response of rat recorded by the grid electrode array with $9 \mathrm{kHz}$ sound stimulation. Measured results shows that localized neurons discharge phenomenon (gamma activities, 30-200 Hz) are recorded by the presented grid electrode array. Three different auditory areas are covered by the grid electrode due to the different pattern observations. The experimental results show that recorded evoked potential on auditory cortex is about $\pm 70 \mathrm{uV}$, and the minimal effective sound stimulation magnitude is $20 \mathrm{~dB}$ SPL.

Next, real 3D neural signal propagation observation in human cerebral resection cortex layers is achieved by practical implantation after resection surgery in cooperation with the Buddhist Tzu-Chi Hospital in Hua-Lien City, Taiwan. All the study procedures follow the IRB rule of Buddhist Tzu-Chi Hospital. A patient suffering from epilepsy seizures needed surgery resection treatment. After the resection surgery, the tissue was put into a prepared artificial cerebrospinal fluid (ACSF) solution in a water bath with $37^{\circ} \mathrm{C}$ temperature control and oxygen supply to keep it alive. Usually the neural activity in resection tissue can last for about 2 hour in the solution. The fabricated 3D probe array was inserted into the brain tissue by a manual 3-axis moving stage. From the recording, we can build a 3D distributed spatial signal plot. Figure 10 illustrates the reconstructed 3D near-field potential distribution recorded by $3 \mathrm{D}$ probe array in brain, which allows further studies for event-related observation.

Figure 10. 3D distributed near-field potential recording by 3D probe array from cerebral resection cortex tissue.

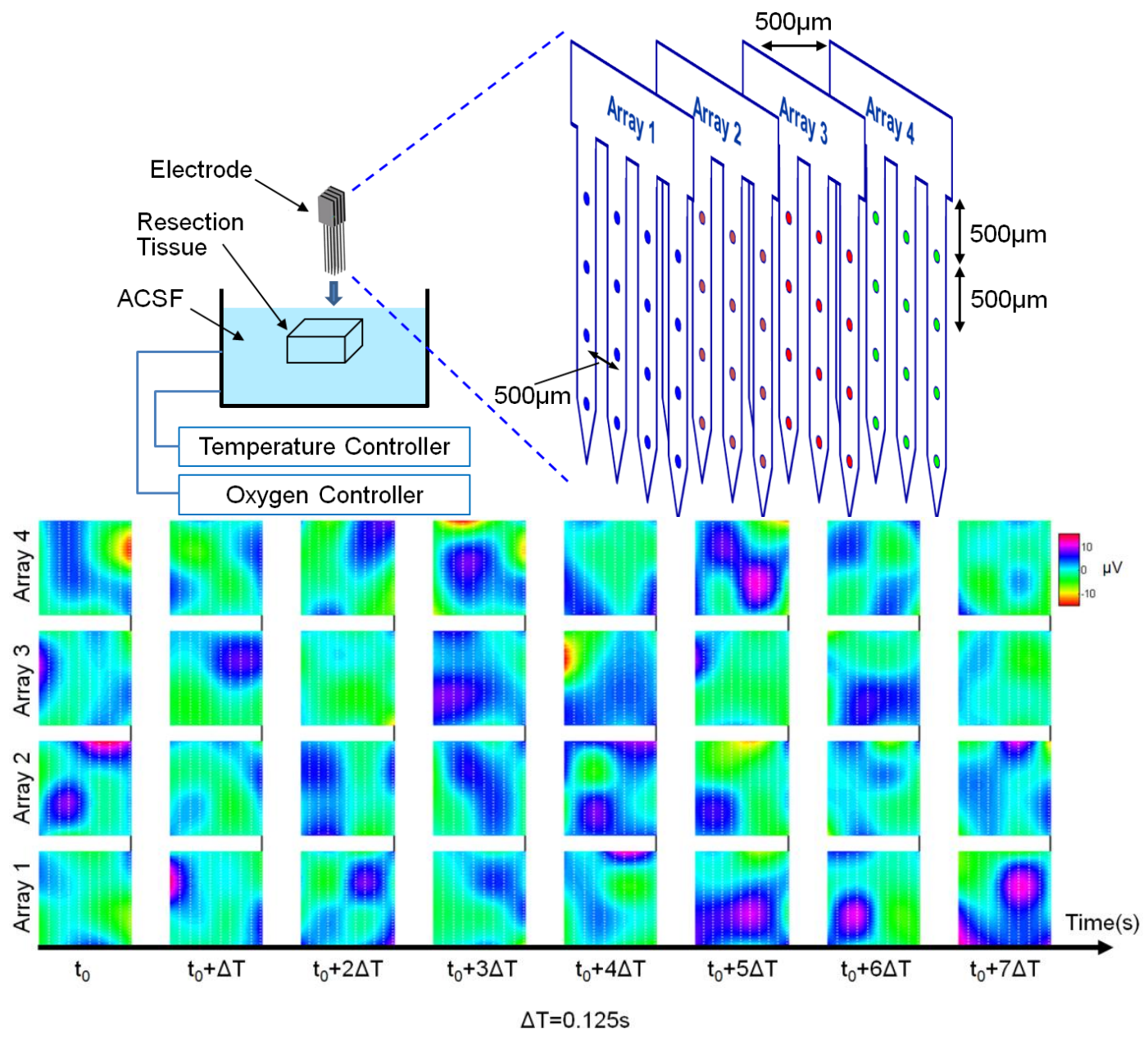


Finally, the present microsystem platform was applied to the recording of pentylenetetrazole (PZT)-induced seizures in rat [27]. Figure 11 shows the photograph of the environment setup. The main board is carried by the backpack on the rat. RF power is generated by a function generator and sent into a customized power amplifier. Power transmission coils connected to the power amplifier are located on the walls of the cage. Recorded data is transferred to a laptop via Bluetooth transmission. Customized Windows-based software provides continuous monitoring and data storage on the laptop.

Figure 11. Induced seizures recording on rat by the present microsystem.

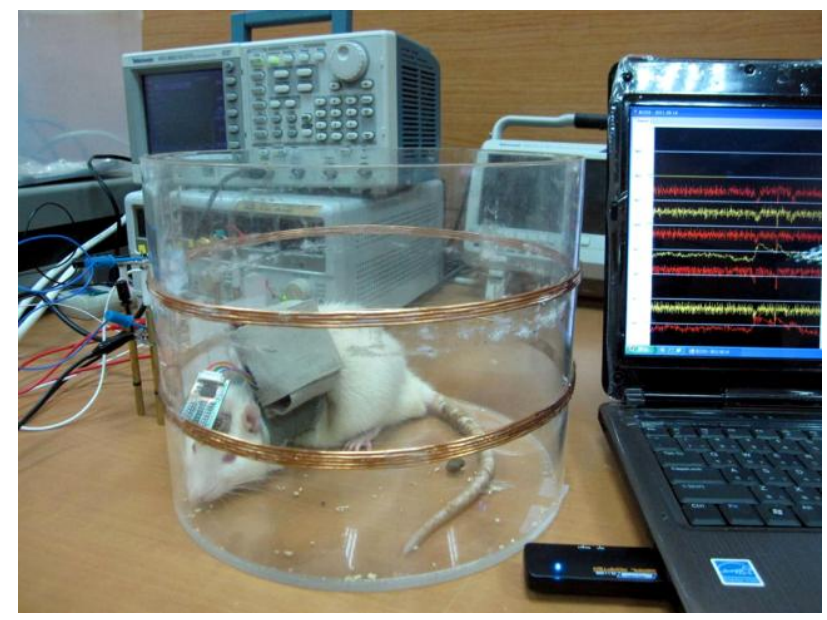

Sprague Dawley rats aged 10-12 weeks and with 500-800 g body weight were used in the experiments. Pentylenetetrazole (PTZ) was prepared fresh daily, dissolved in $0.9 \%$ saline.

After the microsystem was attached to the rat, PZT was administered intraperitoneally in a volume of $10 \mathrm{~mL} / \mathrm{kg}$ body weight. Figure 12 shows the selected four channels located from frontal to parietal region of the cortex. Red dotted rectangles denote the SWD part found in the recording.

Figure 12. Recorded spike-wave (SWD) discharge distributions from frontal to parietal (A) Time domain plot (B) Frequency domain plot. Red dotted rectangle denotes the SWD part founded in the recording.

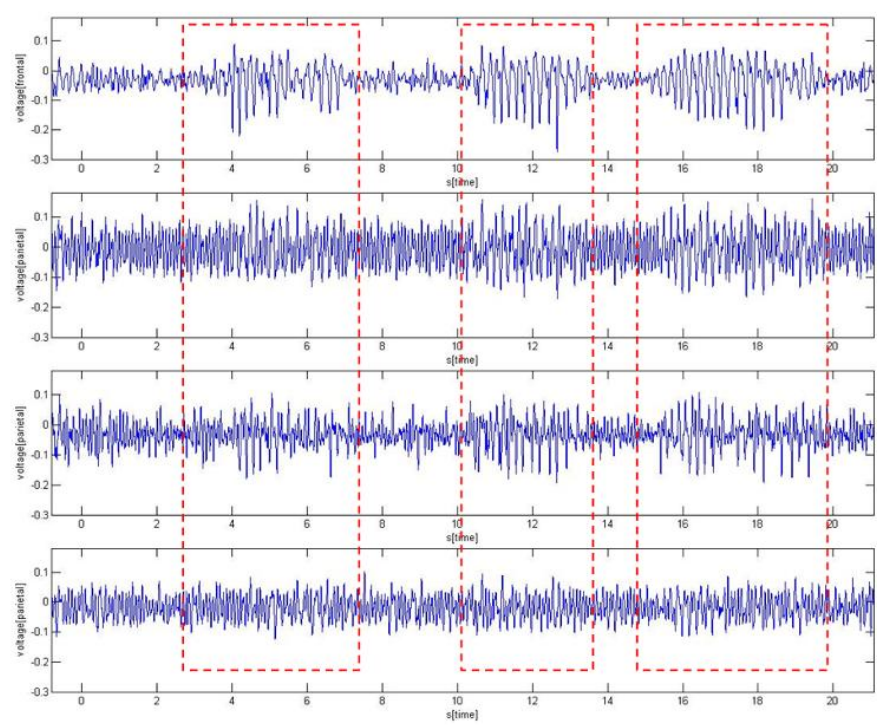

(A) Time domain

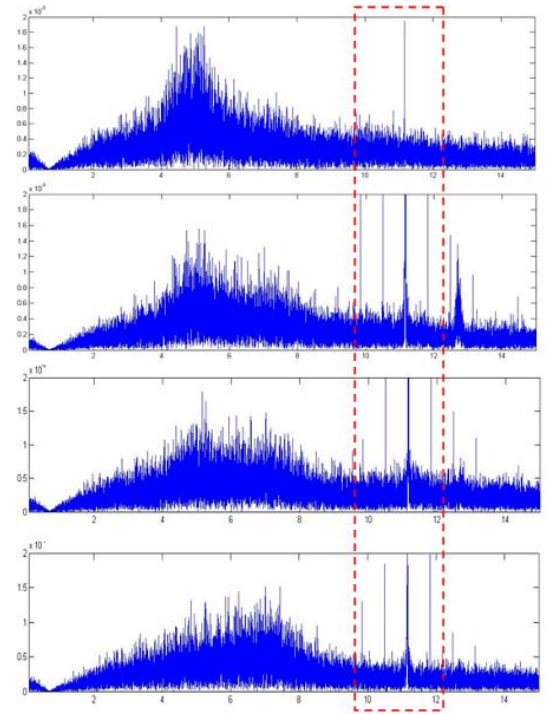

(B) Frequency domain 
From Figure 12(A), it is clear that significant SWD can be found in the frontal zone, and its magnitude is decreased as the recording location is further from the frontal due to the fact that PZT-induced seizures mainly occur in the frontal. Figure 12(B) illustrates the frequency analysis of the recorded data, obvious peaks can be observed in the frequency range of $8-12 \mathrm{~Hz}$ which is the main seizure component range.

\section{Discussion and Conclusions}

In this paper we present a wireless and battery-less system platform and demonstrate it in various implantation applications with different neural sensors. The main contribution of this paper is to integrate different parts, including sensors, customer-designed circuits and commercial chips, into a complete system for neural sensing. The complete system weights $13.17 \mathrm{~g}$, which meets the requirements for being readily carried by rats [28]. Different sensing targets, including ECoG and near-field neural activities, can be monitored by the proposed grid electrode array and 3D array. Each part of the system is designed and characterized in the paper. Practical in vivo recordings including auditory response, brain resection tissue and PZT-induced seizure recordings demonstrate the correct functioning of the proposed system. Currently, some similar works using analog wireless transmission techniques [28,29] are reported for neural sensing applications. In this case, these systems can provide small packaging and lightweight without digital processing circuits, but they may also easily suffer interference from surrounding noise. Highly integrated systems also have been reported for small flying insects [30], hence, to integrate the circuits into monolithic chip will be our next step. Two kinds of electrode design are reported in this paper for measurement on brain surface and in brain tissue. It will be another issue to integrate them into a single sensor to provide observation on and in the brain at the same time.

\section{Acknowledgments}

This work was supported in part by the National Science Council, Taiwan, R.O.C. under Contract No. 101-2220-E-009-030, No. 101-2220-E-009-018, No. 101-2220-E-009-017, and "Aim for the Top University Plan" of the National Chiao Tung University and Ministry of Education, Taiwan. This work was also particularly supported by R\&D Piloting Cooperation Projects between Industries and Academia at Science Parks under Contract Number: 100A20 and the UST-UCSD International Center of Excellence in Advanced Bioengineering sponsored by the Taiwan National Science Council I-RiCE Program under Grant Number: NSC-101-2911-I-009-101. The authors would like to thank National Chip Implementation Center (CIC) for chip fabrication.

\section{References}

1. Schwartz, A.B.; Cui, X.T.; Weber, D.J.; Moran, D.W. Brain-controlled interfaces: Movement restoration with neural prosthetics. Neuron 2006, 52, 205-220.

2. Peng, C.; Chaimanonart, N.; Ko, W.H.; Young, D.J. A Wireless and batteryless 10-bit implantable blood pressure sensing microsystem with adaptive RF powering for real-time laboratory mice monitoring. IEEE J. Solid-State Circuits 2009, 44, 3631-3644. 
3. Yin, M.; Ghovanloo, M. A low-noise clockless simultaneous 32-channel wireless neural recording system with adjustable resolution. Analog. Integr. Circuits Process. 2011, 66, 417-431.

4. Young, C.P.; Liang, S.F.; Chang, D.W.; Liao, Y.C.; Shaw, F.Z.; Hsieh, C.H. A portable wireless online closed-loop seizure controller in freely moving rats. IEEE Trans. Instrum. Meas. 2011, 60, 513-521.

5. Deadwyler, S.A.; Hampson, R.E.; Collins, V. A wireless recording system that utilizes Bluetooth technology to transmit neural activity in freely moving animals. J. Neurosci. Meth. 2009, 182, 195-204.

6. Denisov, A.; Yeatman, E. Ultrasonic vs. Inductive Power Delivery for Miniature Biomedical Implants. In Proceedings of 2010 International Conference on Body Sensor Networks (BSN), London, UK, 7-9 June 2010; pp. 84-89.

7. Stieglitz, T.; Boretius, T.; Ordonez, J.; Hassler, C.; Henle, C.; Meier, W.; Plachta, D.T.T.; Schuettler, M. Miniaturized neural interfaces and implants. Proc. SPIE 2012, 8251, doi:10.1117/12.912526.

8. Chang, C.W.; Chen, Y.J.; Hung, S.H.; Chiou, J.C. A Wireless and Batteryless Microsystem with Implantable Grid Electrode/3-dimensional Probe Array for ECoG and Extracellular Neural Recording on Rat. In Proceedings of 2011 16th International Solid-State Sensors, Actuators and Microsystems Conference (TRANSDUCERS), Beijing, China, 5-9 June 2011; pp. 2176-2179.

9. Sun, T.; Park, W.T.; Cheng, M.Y.; An, J.Z.; Xue, R.F.; Tan, K.L.; Je, M. Implantable polyimide cable for multichannel high-data-rate neural recording microsystems. IEEE Trans. Biomed. Eng. 2012, 59, 390-399.

10. Chang, C.-W.; Hou, K.-C.; Shieh, L.-J.; Hung, S.-H.; Chiou, J.-C. Wireless powering electronics and spiral coils for implant microsystem toward nanomedicine diagnosis and therapy in free-behavior animal. Solid-State Electron. 2012, 77, 93-100.

11. Vaillancourt, P.; Djemouai, A.; Harvey, J.F.; Sawan, M. EM Radiation Behavior upon Biological Tissues in a Radio-Frequency Power Transfer Link for a Cortical Visual Implant. In Proceedings of the 19th Annual International Conference of the IEEE Engineering in Medicine and Biology Society, Chicago, IL, USA, 30 October-2 November 1997; Voume 6, pp. 2499-2502.

12. Chang, C.W.; Chiou, J.C. Development of a three dimensional neural sensing device by a stacking method. Sensors 2010, 10, 4238-4252.

13. Wise, K.D.; Sodagar, A.M.; Yao, Y.; Gulari, M.N.; Perlin, G.E.; Najafi, K. Microelectrodes, microelectronics, and implantable neural microsystems. Proc. IEEE 2008, 96, 1184-1202.

14. Bai, Q.; Wise, K.D. Single-unit neural recording with active microelectrode arrays. IEEE Trans. Biomed. Eng. 2001, 48, 911-920.

15. Merriam, S.M.E.; Srivannavit, O.; Gulari, M.N.; Wise, K.D. A Three-Dimensional 64-Site Folded Electrode Array Using Planar Fabrication. J. Microelectromech. Syst. 2011, 20, 594-600.

16. Yao, Y.; Gulari, M.N.; Wiler, J.A.; Wise, K.D. A microassembled low-profile three-dimensional microelectrode array for neural prosthesis applications. J. Microelectromech. Syst. 2007, 16, 977-988.

17. Rubehn, B.; Bosman, C.; Ostenveld, R.; Fries, P.; Stieglitz, T. A MEMS-based flexible multichannel ECoG-electrode array. J. Neural. Eng. 2009, 6, 036003. 
18. Finkenzeller, K. RFID Handbook-Fundamentals and Applications in Contactless Smart Cards and Identification, 2nd ed.; John Wiley \& Sons: West Sussex, UK, 2003.

19. Kendir, G.A.; Liu, W.T.; Wang, G.X.; Sivaprakasam, M.; Bashirullah, R.; Humayun, M.S.; Weiland, J.D. An optimal design methodology for inductive power link with class-E amplifier. IEEE Trans. Circuits Syst. I 2005, 52, 857-866.

20. Jow, U.M.; Ghovanloo, M. Design and optimization of printed spiral coils for efficient transcutaneous inductive power transmission. IEEE Trans. Biomed. Circuits Syst. 2007, 1, 193-202.

21. Jow, U.M.; Ghovanloo, M. Modeling and optimization of printed spiral coils in air, saline, and muscle tissue environments. IEEE Trans. Biomed. Circuits Syst. 2009, 3, 339-347.

22. Ko, W.; Liang, S.; Fung, C. Design of radio-frequency powered coils for implant instruments. Med. Biol. Eng. Comput. 1977, 15, 634-640.

23. Harrison, R.R. Designing Efficient Inductive Power Links for Implantable Devices. In Proceedings of IEEE International Symposium on Circuits and Systems, ISCAS 2007, New Orleans, LA, USA, 27-20 May 2007; pp. 2080-2083.

24. Kurs, A.; Karalis, A.; Moffatt, R.; Joannopoulos, J.D.; Fisher, P.; Soljacic, M. Wireless power transfer via strongly coupled magnetic resonances. Science 2007, 317, 83-86.

25. Rincon-Mora, G.A.; Allen, P.E. A low-voltage, low quiescent current, low drop-out regulator. IEEE J. Solid-State Circuits 1998, 33, 36-44.

26. Seese, T.M.; Harasaki, H.; Saidel, G.M.; Davies, C.R. Characterization of tissue morphology, angiogenesis, and temperature in the adaptive response of muscle tissue to chronic heating. Lab. Invest. 1998, 78, 1553-1562.

27. Depaulis, A.; Vergnes, M.; Marescaux, C. Endogenous control of epilepsy-The nigral inhibitory system. Prog. Neurobiol. 1994, 42, 33-52.

28. Szuts, T.A.; Fadeyev, V.; Kachiguine, S.; Sher, A.; Grivich, M.V.; Agrochao, M.; Hottowy, P.; Dabrowski, W.; Lubenov, E.V.; Siapas, A.G.; et al. A wireless multi-channel neural amplifier for freely moving animals. Nat. Neurosci. 2011, 14, U263-U363.

29. Roy, S.; Wang, X. Wireless multi-channel single unit recording in freely moving and vocalizing primates. J. Neurosci. Meth. 2012, 203, 28-40.

30. Thomas, S.J.; Harrison, R.R.; Leonardo, A.; Reynolds, M.S. A battery-free multichannel digital neural/emg telemetry system for flying insects. IEEE Trans. Biomed. Circuits Syst. 2012, 6, 424-436.

(C) 2013 by the authors; licensee MDPI, Basel, Switzerland. This article is an open access article distributed under the terms and conditions of the Creative Commons Attribution license (http://creativecommons.org/licenses/by/3.0/). 